\title{
AVALIAÇÃO DO CONHECIMENTO \\ NUTRICIONAL DE MULHERES FISICAMENTE \\ ATIVAS E SUA ASSOCIAÇÃO COM O ESTADO NUTRICIONAL
}

\author{
ESP. NATÁLIA MORI GONÇALVES DE CASTRO \\ Especialista em nutrição desportiva e qualidade de vida pelas \\ Faculdades Integradas de Santo André (Fefisa) (São Paulo - Brasil) \\ E-mail: natpatota@hotmail.com
}

\begin{abstract}
ESP. MURILO DÁTTILO
Especialista em nutrição desportiva e qualidade de vida pelas Fefisa (São Paulo - Brasil)

Mestrando pelo Departamento de Psicobiologia da Universidade Federal de São Paulo (UNIFESP)

(São Paulo - Brasil)

E-mail: murilo@cepebr.org
\end{abstract}

\section{DRA. LAURA CUVELLO LOPES}

Doutora em ciências pela Unifesp

Professora das áreas de educação e saúde do Centro Universitário Ítalo Brasileiro

Professora do curso de pós-graduação em nutrição desportiva e qualidade de vida das Fefisa

(São Paulo - Brasil)

E-mail: Icuvello@hotmail.com

\section{RESUMO}

O objetivo do presente estudo foi avaliar o conhecimento nutricional de mulheres fisicamente ativas e correlacionar a pontuação obtida com variáveis antropométricas e nível de escolaridade. A amostra foi composta por 60 mulheres e os dados antropométricos mensurados foram massa corporal, estatura, Índice de Massa Corporal (massa corporal/estatura²) e perímetro da cintura. A amostra apresentou conhecimento nutricional moderado e correlações significativas foram obtidas da pontuação do questionário de conhecimento nutricional com o Índice de Massa Corporal ( $r=-0,38, p<0,05)$, perímetro da cintura $(r=-0,30, p<$ $0,05)$ e nível de escolaridade $(r=0,36, p<0,05)$. O conhecimento nutricional pode estar associado com o estado nutricional e o nível de escolaridade.

PALAVRAS-CHAVE: Estado nutricional; conhecimento nutricional; exercício físico e escolaridade.

* O presente trabalho não contou com apoio financeiro de nenhuma natureza e tampouco houve conflitos de interesses para sua realização. 


\section{INTRODUÇÃO}

Nos dias atuais, o número de indivíduos apresentando quadros de sobrepeso e obesidade vem demonstrando crescente aumento, o que tem promovido preocupação de muitos países devido ao grande risco à saúde que esses quadros podem trazer (PereIRA et al., 2003). Atualmente, estimativas indicam que I , I bilhão de adultos da população mundial apresenta Índice de Massa Corporal (IMC) $\geq 25,0$ $\mathrm{kg} / \mathrm{m}^{2}$, enquanto 312 milhões se encontram na faixa de obesidade (IMC $\geq 30,0 \mathrm{~kg} /$ $\mathrm{m}^{2}$ ) (Hossaln et al., 2007), sendo esta já considerada uma epidemia mundial (POPKIN; DOAK, 1998; WHO, 2000).

De fato, muitos fatores estão implicados no aumento de massa corporal, mas ainda se sustenta a hipótese de que a marcante redução do nível de atividade física associada ao aumento do consumo energético são os principais responsáveis (MUST, 1996). Em função da estreita relação entre os hábitos alimentares e o valor energético consumido que, em conjunto, são capazes de influenciar o balanço energético, métodos para avaliação do consumo alimentar vêm sendo colocado em destaque nas pesquisas científicas com o intuito de identificar o padrão dietético e, por fim, propor modificações nos hábitos alimentares diários (Monteiro et al, 2000).

Dentre esses métodos, podem-se citar tanto ferramentas objetivas (por ex.: registro alimentar de três dias não consecutivos) quanto subjetivas, com destaque para o Questionário de Conhecimento Nutricional (QCN). Vem sendo postulado que essa ferramenta, criada para avaliar o processo cognitivo individual relacionado à alimentação e nutrição (AXELSON; BRINBERG, I992; SCAGLIUSI et al., 2006), pode ser capaz de influenciar a seleção alimentar (SCAGLIUsı et al., 2006) e com o sucesso na prevenção de doenças crônicas não transmissíveis (DESPRÉS; LAMARCHE, 1994). Além disso, outras pesquisas têm buscado identificar o papel do conhecimento nutricional no estado nutricional, mas os resultados não têm sido favoráveis (DÁTtıo et al., 2009; O'BRIEN; DaVIES, 2007).

Curiosamente, três aspectos estão associados com um aprimoramento do nível de conhecimento nutricional: a prática de exercícios físicos (CUPISTI et al., 2002), indivíduos do gênero feminino (DATTILO et al., 2009; NicAsTro et al., 2008) e nível de escolaridade (DATTLO et al., 2009). O primeiro pode estar associado com uma busca por hábitos alimentares mais saudáveis, visto que o exercício físico é considerado como tal, enquanto o segundo pode ser justificado pela constante busca pela melhora do perfil estético (ZAWILA et al., 2003). Além disso, o nível de escolaridade parece ser um dos fatores mais básicos para todo o processo educa- 
tivo do indivíduo, proporcionando um maior grau de discernimento e busca por informações. Sendo assim, o presente trabalho teve como objetivos avaliar o nível de conhecimento nutricional de mulheres fisicamente ativas de uma academia de ginástica do município de São Bernardo do Campo e correlacionar esse conhecimento com o estado nutricional e escolaridade.

\section{MATERIAL E MÉTODOS}

\section{AMOSTRA}

O presente estudo caracteriza-se como transversal, sendo a amostra composta por 60 mulheres, com idade entre 20 e 60 anos, praticantes de exercícios físicos monitorados em uma academia só para mulheres do município de São Bernardo do Campo, São Paulo. Como critério de inclusão foi considerado somente mulheres acima de 18 anos e que praticassem exercícios físicos regulares por pelo menos um ano ininterrupto.

O convite foi realizado verbalmente e, após esclarecimentos sobre os procedimentos, objetivos e riscos, elas assinaram um Termo de Consentimento Livre e Esclarecido. O presente estudo obteve aprovação do Comitê de Ética e Pesquisa das Faculdades Integradas de Santo André, sob protocolo n. $122 / 09$.

\section{PROCEDIMENTOS}

Após a realização do convite e a assinatura do Termo de Consentimento Livre e Esclarecido, os indivíduos eram acompanhados até uma sala especialmente reservada para a avaliação antropométrica, a qual foi criteriosamente realizada antes do início de qualquer tipo de exercício físico. Após essa etapa, foi realizada a orientação quanto ao preenchimento do QCN, que poderia ser realizado antes ou após a prática das atividades no estabelecimento, de acordo com a disponibilidade de tempo de cada voluntária. O questionário foi autoaplicado e as voluntárias orientadas a responder da maneira mais honesta possível. Decidiu-se não reaplicar o questionário, pois estas poderiam receber alguma educação nutricional nos próximos dias.

\section{AVALIAÇÃO ANTROPOMÉTRICA}

A massa corporal $(\mathrm{kg})$ foi aferida por meio de uma balança fixa da marca Micheletti, com precisão de 0,05 gramas e capacidade máxima de $150 \mathrm{~kg}$. A 
estatura foi aferida por meio de um estadiômetro padrão, seguindo a diretriz da International Society of Advancement in Kinanthropometry (MARFELL-JONES et al., 2006). O IMC foi calculado pela razão entre massa corporal $(\mathrm{kg})$ e estatura ao quadrado $\left(\mathrm{m}^{2}\right)$, sendo os indivíduos classificados segundo os critérios propostos pela Organização Mundial de Saúde (WHO, 2000). O perímetro da cintura (cm) foi mensurado com uma fita métrica da marca Sanny ${ }^{\circledR}$, sendo a medida realizada no ponto médio entre a crista ilíaca e o último arco costal.

\section{AVALIAÇÃO DO NÍVEL DE CONHECIMENTO NUTRICIONAL}

A escala escolhida para mensuração do conhecimento nutricional neste estudo foi a desenvolvida por Harnack et al. ( 1997) e, posteriormente, traduzida, adaptada e validada para o Brasil por Scagliusi et al. (2006). A versão original elaborada por Harnack et al. (idem) foi uma ferramenta utilizada para avaliação de indivíduos adultos no National Health Interview Survey Cancer Epidemiology, em 1992, com o intuito de testar se o conhecimento nutricional sobre prevenção de câncer se correlacionava com práticas alimentares que, realmente, prevenissem a doença. Nesse questionário, os itens que o compunham estavam estruturados em três partes, as quais buscavam avaliar: I) a relação entre dieta e doença; 2) quantidade de fibras e lipídeos nos alimentos e; 3) as recomendações de ingestão de frutas e hortaliças. Como esse questionário foi elaborado com tópicos pertinentes ao padrão dietético americano, Scagliusi et al. (idem) propuseram a tradução, adaptação e, por fim, validação de seu conteúdo para a população brasileira, com estudantes de nutrição e pacientes com transtornos alimentares. Nesse trabalho, após todas as etapas propostas, constatou-se que a ferramenta apresentou nível de precisão modesto, representado pelo coeficiente de Spearman $r=0,52$.

De acordo com Harnack et al. (idem) e Scagliusi et al. (idem), os critérios para classificação da pontuação foram: pontuações totais entre zero e seis indicam baixo conhecimento nutricional, entre sete e dez indicam moderado conhecimento nutricional e acima de dez indicam alto conhecimento nutricional.

\section{NÍVEL DE ESCOLARIDADE}

O nível de escolaridade foi determinado a partir de uma questão aberta, expressa em anos. 
As análises foram conduzidas pelo software Statistica versão 6.0 (Statisoft $\left.{ }^{\circledR}\right)$. Foi utilizado o teste de Pearson para as correlações entre a pontuação do QCN e IMC, perímetro da cintura e nível de escolaridade. O teste t de Student para uma amostra foi utilizado para comparação da pontuação obtida em nossa amostra com a pontuação já descrita em um trabalho previamente publicado, sendo esse dado demonstrado na seção "Discussão". Todos os valores são expressos em média \pm desvio-padrão, com nível de significância de $p \leq 0,05$.

\section{RESULTADOS}

As características da amostra estão representadas na tabela I e cabe ressaltar que, dentro dos critérios de inclusão eleitos, não houve nenhuma recusa para participação na pesquisa. Foi possível observar que, para o IMC, a amostra se apresentou na faixa de sobrepeso de acordo com os pontos de corte da $\mathrm{WHO}$ (2000), enquanto o perímetro da cintura indicou risco aumentando para obesidade associada a complicações metabólicas, seguindo os critérios propostos por Hasselmann et al. (2008). Outro ponto a ser levado em consideração é a pontuação média obtida no QCN, indicando nível moderado de conhecimento nutricional.

Tabela I - Características da amostra

\begin{tabular}{|l|c|c|c|}
\hline \multicolumn{1}{|c|}{ Variáveis } & M \pm DP & Mínimo & Máximo \\
\hline Idade (anos) & $40 \pm 12$ & 22 & 68 \\
\hline Massa corporal $(\mathrm{kg})$ & $71,0 \pm 12,0$ & 51,0 & 106,3 \\
\hline Estatura $(\mathrm{m})$ & $1,6 \pm 0,1$ & 1,5 & 1,7 \\
\hline IMC $\left(\mathrm{kg} / \mathrm{m}^{2}\right)$ & $27,0 \pm 4,5$ & 20,1 & 40,6 \\
\hline Perímetro da cintura (cm) & $94,7 \pm 12,8$ & 70,3 & 130,5 \\
\hline Prática de EF (anos) & $4,2 \pm 6,3$ & 1 & 20 \\
\hline QCN (pontuação) & $8,3 \pm 1,7$ & 4 & 11 \\
\hline Nível de escolaridade (anos) & $14,0 \pm 2,5$ & 8,0 & 17,0 \\
\hline
\end{tabular}

Abreviaturas: IMC = Índice de Massa Corporal, EF = exercício físico, QCN = Questionário de Conhecimento Nutricional.

Na figura I está representada a distribuição, em número e percentual, do nível de conhecimento nutricional das voluntárias, de acordo com os pontos de corte baixo (16\%), moderado (77\%) e alto (7\%) de conhecimento nutricional. 
De modo geral, é possível observar que a grande maioria apresentou nível de conhecimento moderado.

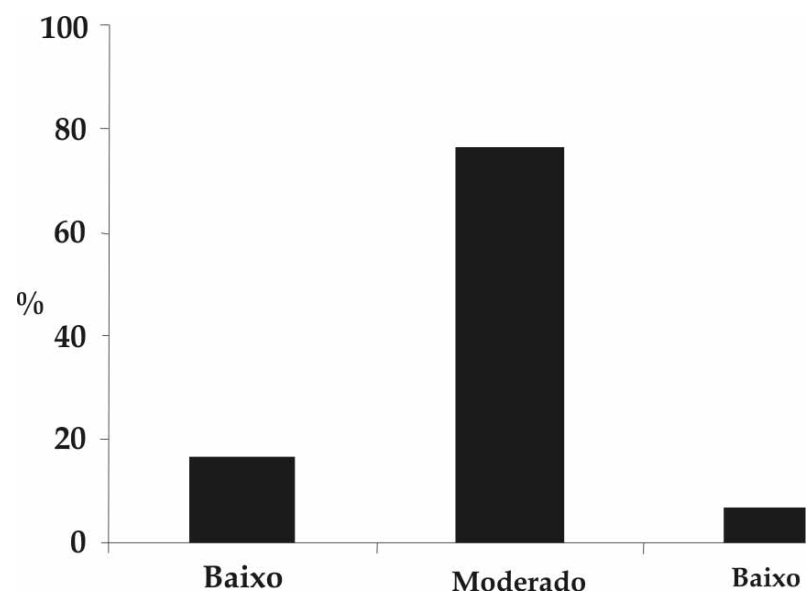

Figura I: Distribuição, em número e em porcentagem, de acordo com a classificação obtida no QCN.

Nas figuras 2 e 3 estão representadas correlações entre a pontuação do QCN e IMC e perímetro de cintura respectivamente. É possível observar que, para ambas as análises, a pontuação do QCN apresentou correlação negativa, estatisticamente significativa.

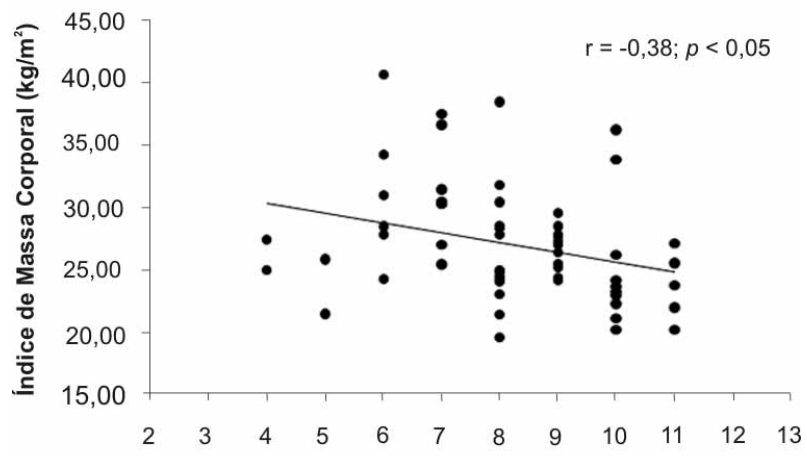

Figura 2: Correlação entre IMC e pontuação do QCN. 


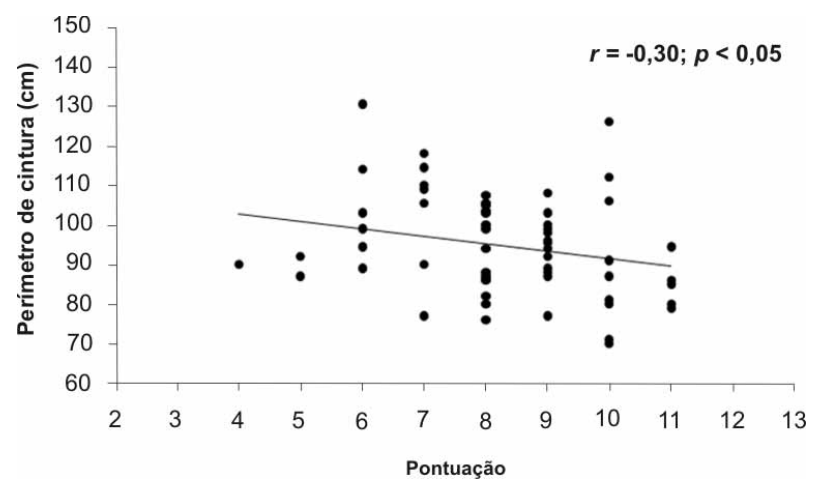

Figura 3: Correlação entre perímetro da cintura e pontuação do QCN.

Quando realizada a correlação entre a pontuação do QCN com o nível de escolaridade, foi possível observar que essas duas variáveis apresentaram uma correlação positiva, estatisticamente significativa (Figura 4).

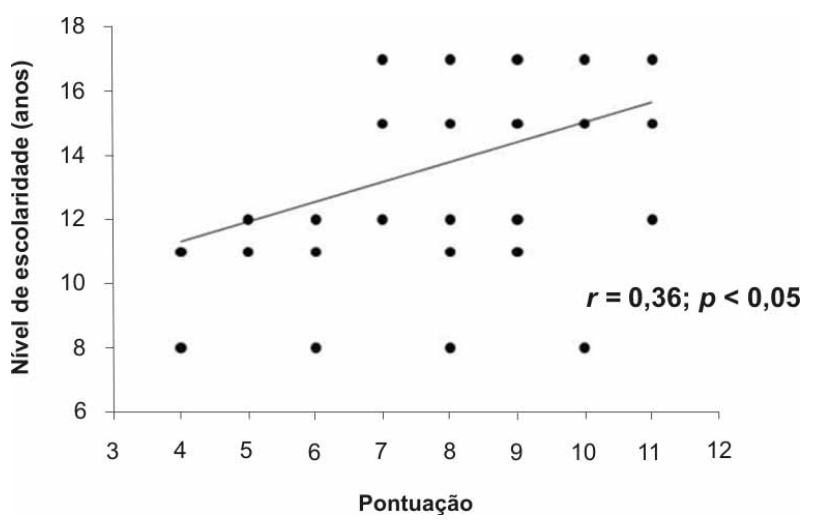

Figura 4: Correlação entre nível de escolaridade e pontuação do QCN.

\section{DISCUSSÃO}

A avaliação do nível de conhecimento nutricional vem ganhando espaço entre as pesquisas científicas nos últimos anos por causa da premissa de que o conhecimento sobre nutrição e alimentação pode estar associado com práticas alimentares (DatTILO et al., 2009; NicAstro et al., 2008; RAstMANESCH et al., 2007; RAYMOND- 
BARKER et al., 2007; CUPISTI et al., 2002; BURKE, 1995). Entretanto, poucos trabalhos utilizaram a presente escala para avaliação do conhecimento nutricional. Dentre eles, Scagliusi et al. (2006) avaliaram mulheres com transtornos alimentares e estudantes de nutrição e, segundo os autores, pacientes com transtornos alimentares obtiveram pontuação de 7,4 \pm 2,6, enquanto, no grupo das estudantes, a pontuação foi 1 0,5 \pm I,7 ( $p=0,000000$ I). No estudo realizado por Freitas et al. (2006), verificou-se que mulheres vegetarianas apresentaram conhecimento nutricional superior a mulheres onívoras ( $10,6 \pm 1,7$ versus 8,7 $\pm 2,3$ respectivamente, $p=0,0001)$.

Até o momento, o presente trabalho é o terceiro publicado envolvendo a aplicação desse mesmo questionário em indivíduos fisicamente ativos e atletas, com o intuito específico de avaliar o conhecimento sobre nutrição, alimentação e saúde. O primeiro, publicado por Nicastro et al. (2008), avaliou atletas profissionais e amadoras de atletismo e, dentre os dados encontrados, verificou-se que o público amador (composto exclusivamente por mulheres) apresentou pontuação significantemente superior ao dos atletas profissionais $(8,9 \pm 1,7$ versus 7,3 $\pm 1,8$, $p<0,0$ I). O segundo trabalho foi realizado por Oliveira et al. (2009), envolvendo a avaliação de atletas profissionais de judô, no qual os autores não evidenciaram diferenças significantes entre as pontuações de homens e mulheres $(6,9 \pm 2,0$ versus $6,9 \pm 1,7, p=0,56$ ).

A estruturação da escala utilizada é baseada em faixas de pontuação que permitem a devida classificação do nível de conhecimento nutricional da amostra. Entretanto, é pertinente que seja realizada a análise da pontuação obtida, pois, embora o elevado conhecimento nutricional seja considerado com I I e 12 pontos, o nível moderado apresenta uma variação muito mais ampla. No presente trabalho, a amostra demonstrou nível de conhecimento nutricional moderado $(8,3 \pm 1,7)$ e, curiosamente, foi significantemente maior ao obtido por Dattilo et al. (2009) em mulheres sedentárias $(t=2,92, g l=59, p<0,0$ l). Possivelmente, fatores como a escolaridade, o nível socioeconômico e a prática de exercícios físicos possam ser importantes justificativas para essa maior pontuação.

No presente estudo foi possível observar que correlações negativas significantes foram obtidas entre a pontuação do QCN e IMC (Figura 2) e perímetro da cintura (Figura 3), sugerindo que o conhecimento nutricional parece possuir um efeito protetor contra o aumento de massa corporal. Entretanto, cabe mencionar que este parece ser somente um fator contribuinte, pois a amostra apresentou IMC dentro do ponto de corte para sobrepeso. Embora não tenha sido realizada a avaliação do consumo alimentar das voluntárias, evidências indicam que o conhecimento nutricional pode estar associado com a seleção alimentar (SCAGLIUSI 
et al., 2006) e, dessa forma, favorecer hábitos alimentares saudáveis e prevenir o aumento de massa corporal.

Em contrapartida, outros trabalhos presentes na literatura apresentam dados divergentes a respeito da tríade conhecimento nutricional, hábitos alimentares e estado nutricional. O'Brien e Davies (2007) avaliaram 500 indivíduos adultos por meio de um questionário de conhecimento nutricional e verificaram que o alto nível de conhecimento não se correlacionou com o IMC, indicando que esse pode não ser o fator mais importante para que indivíduos com excesso de massa corporal adotem hábitos saudáveis. Da mesma forma, Montero Bravo et al. (2006), avaliando I 05 estudantes universitários, verificaram que, mesmo quando os alunos acreditavam ter um bom conhecimento nutricional, este não estava necessariamente associado com modificações dos hábitos alimentares. Já Dattilo et al. (2009), avaliando 42 pacientes de um centro de reabilitação do município de São Paulo, não identificaram correlações significativas do conhecimento nutricional com o IMC, evidenciando que o conhecimento nutricional parece, de fato, não estar diretamente relacionado com modificações dos hábitos alimentares.

Um dos principais determinantes do conhecimento nutricional parece ser a formação escolar do indivíduo e, corroborando outros dados (DATTILO et al., 2009; OBAYASH et al., 2003; SAPP; JENSEN, 1997), observamos uma correlação positiva entre o nível de conhecimento nutricional com o grau de escolaridade. Dessa forma, embora o nível de escolaridade não seja um contribuinte direto para aumento do conhecimento específico sobre alimentação e nutrição, em função de não serem ensinados conteúdos estruturados a respeito do assunto em longo prazo, o período escolar pode ser determinante no aprimoramento do grau de discernimento do indivíduo. Assim, a busca por informações e a tomada de decisão para determinadas atitudes podem ser indiretamente favorecidas pelo nível de escolaridade e, como consequência, se tornar componente essencial na obtenção de conhecimentos associados à nutrição e alimentação.

\section{CONCLUSÃO}

Foi possível concluir que a amostra avaliada apresentou conhecimento nutricional moderado, verificando-se uma importante correlação negativa deste conhecimento com o IMC e perímetro da cintura. $O$ presente trabalho indica que o aprimoramento do conhecimento sobre nutrição e alimentação pode ser uma das ferramentas para prevenção e tratamento da obesidade. Destacamos ainda o papel da educação escolar como capaz de influenciar o conhecimento a respeito de informações sobre alimentação e nutrição. 
Assessment of nutrition knowledge of women physically active and its relationship with nutritional status

ABSTRACT: The aim of the present study was to evaluate nutritional knowledge of women physically active and correlate it with anthropometric variables and educational level. The sample was composed by 60 women. The anthropometric variables measured were body weight, height, Body Mass Index (body weight/height ${ }^{2}$ ), waist circumference and nutritional knowledge. The sample demonstrated moderate nutritional knowledge and the questionnaire score was correlated with Body Mass Index ( $r=-0,38, p<0,05)$, waist circumference $(r=-0,30, p<0,05)$ and educational level $(r=0,36, p<0,05)$. The nutritional knowledge may be associated with nutritional and educational status.

KEY WORDS: Nutritional status; nutritional knowledge; physical exercise and educational status.

\section{Evaluación de conocimientos de nutrición de las mujeres físicamente activas y su relación con el estado nutricional}

RESUMEN: El objetivo de este estudio fue evaluar el conocimiento nutricional de las mujeres que practican actividad física y correlacionar los resultados obtenidos con variables de la antropometría. La muestra comprende 60 personas y las variables analizadas son: Peso, estatura, el cálculo del Índice de Masa Corporal, perímetro de la cintura y conocimiento nutricional. Se observó que, en general, ha evaluado el conocimiento nutricional moderado. Sin embargo, hubo correlación significativa entre la puntuación del cuestionario con el índice de masa corporal $(r=-0,38)$, perímetro de la cintura $(r=-0,30)$ y nivel educativo $(r=0,36)$. El conocimiento nutricional puede estar asociada con el estado nutricional y educativo.

PALABRAS CLAVES: Estado nutricional; conocimiento nutricional; ejercicio físico e nivel de instrucción.

\section{REFERÊNCIAS}

AXELSON, M.; BRINBERG, D. The measurement and conceptualization of nutrition knowledge. Journal Nutrition Education, v. 24, n. 5, p. 239-246, set.-out. 1992.

BURKE, L. Practical issues in nutrition for athletes. Journal of Sports Sciences, v. I3, n. SI, p. S83-S90, 1995.

CUPISTI, A.; D'ALESSANDRO, C.; CASTROGIOVANNI, S.; BARALE, A.; MORELLI, E. Nutrition knowledge and dietary composition in Italian adolescent female athletes and nonathletes. Int J Sport Nutr Exerc Metab, Champaign, v. I2, n. 2, p. 207-2 19, jun. 2002.

DATTILO, M.; FURLANETTO, P.; KURODA, A. P.; NICASTRO, H; COIMBRA, P. C. F. C.; SIMONY, R. F. Conhecimento nutricional e sua associação com o índice de massa corporal. Nutrire: Revista da Sociedade Brasileira de Alimentação e Nutrição, São Paulo, v. 34, n. I, p. 75-84, abr. 2009. 
DESPRÉS, J. P.; LAMARCHE, B. Low intensity endurance exercise training, plasma lipoporotein and the risk of coronary heart disease. Journal of Internal Medicine, v. 236, n. I, p. 7-22, jul. 1994.

FREITAS, E. C. B.; ALVARENGA, M. S.; SCAGLIUSI, F. B. Avaliação do conhecimento nutricional e frequência de ingestão de grupos alimentares em vegetarianos e não vegetarianos. Revista Brasileira de Nutrição Clinica, v. 2 I , n. 4, p. 267-272, out.-nov.-dez. 2006.

HARNACK, L.; BLOCK, G.; SUBAR, A.; LANE, S.; BRAND, R. Association of cancer prevention-related nutrition knowledge, beliefs, and attitudes to cancer prevention dietary behavior. Journal of the American Dietetic Association, v. 97, n. 9, p. 957-965, set. 1997.

HASSELMANN, H. M.; FAERSTEIN, E.; WERNECK, G. L.; CHOR, D.; LOPES, C. S. Associação entre circunferência abdominal e hipertensão arterial em mulheres: estudo pró-saúde. Caderno de Saúde Pública, v. 24, n. 5, p. I. I87-1.191, maio 2008.

HOSSAIN, P.; KAWAR, B.; EL NAHAS, M. Obesity and diabetes in the developing world - A growing challenge. The New England Journal of Medicine, v. 356, n. 3, p. 213-2 I , jan. 2007.

MARFELL-JONES, M.; OLDS, T.; STEWART, A.; CARTER, L. International standards for anthropometric assessment. Potchefstroom: Isak, 2006.

MONTEIRO, C. A.; MONDINI, L.; COSTA, R. B. L. Mudanças na composição e adequação nutricional da dieta familiar nas áreas metropolitanas do Brasil (1988-1996). Revista de Saúde Pública, v. 34, n. 3, p. 25I-258, jun. 2000.

MONTERO BRAVO, A.; UBEDA MARTÍN, N.; GARCÍA GONZÁLEZ, A. Evaluation of dietary habits of a population of university students in relation with their nutritional knowledge. Nutricion Hospitalaria, v. 2 I, n. 4, p. 466-473, jul.-ago. 2006.

MUST, A. Morbidity and mortality associated with elevated body weight in children and adolescents. The American Journal of Clinical Nutrition, v. 63, n. 3, p. 445S-447S, mar. 1996, Suplemento.

NICASTRO, H.; DATTILO, M.; SANTOS, T. R.; PADILHA, H. G. V.; ZIMBERG, I. Z.; CRISPIM, C. A.; STULBACH, T. E. Aplicação da Escala de Conhecimento Nutricional em atletas profissionais e amadores de atletismo. Revista Brasileira de Medicina do Esporte, v. I4, n. 3, p. 205-208, maio-jun. 2008.

O'BRIEN, G.; DAVIES, M. Nutrition knowledge and body mass index. Health Education Research, v. 22, n. 4, p. 57I-755, ago. 2007.

OBAYASHI, S.; BIANCHI, L. J.; SONG, W. O. Reliability and validity of nutrition knowledge, social-psychological factors, and food label use scales from the 1995 Diet and Health Knowledge Survey. Journal of Nutrition Education and Behavior, v. 35, n. 2, p. 83-91, mar.-abr. 2003.

OLIVEIRA, F. L.; RUSSO, F. M.; MENEGATTI, I.; TOYA, M. M.; STULBACK, T. E.; GARCIA, L. S.; PERON, A. N.; DATTILO, M. Avaliação do conhecimento nutricional de atletas profissionais de judô. Lecturas Educación Física y Deportes, v. I4, n. 138, p. I, nov. 2009. 
PEREIRA, L. O.; FRANCISCHI, R. P.; LANCHA JR., A. H. Obesidade: hábitos nutricionais, sedentarismo e resistência à insulina. Arquivos Brasileiros de Endocrinologia e Metabologia, v. 47, n. 2, p. II I-127, abr. 2003.

POPKIN, B. M.; DOAK, C. M. The obesity epidemic is a worldwide phenomenon. Nutrition Reviews, v. 56, n. 4, p. 106-||4, abr. 1998.

RASTMANESH, R.; TALEBAN, F.A.; KIMIAGAR, M. MEHRABI, Y.; SALEHI, M. Nutritional knowledge and attitudes in athletes with physical disabilities. Journal of Athletic Training, v. 42, n. I, p. 99-105, jan.-mar. 2007.

RAYMOND-BARKER, P.; PETROCZI, A.; QUESTED, E. Assessment of nutritional knowledge in female athletes susceptible to the Female Athlete Triad syndrome. Journal of Occupational Medicine and Toxicology, v. 2, p. 10, set. 2007.

SANTOS, R.; NUNES, A.; RIBEIRO, J. C.; SANTOS, P.; DUARTE, J. A. R.; MOTA, J. Obesidade, síndrome metabólica e atividade física: estudo exploratório realizado com adultos de ambos os sexos, da ilha de S. Miguel, região autônoma dos Açores, Portugal. Revista Brasileira de Educação Física e Esporte, v. 19, n. 4, p. 317-28, out.-dez. 2005.

SAPP, S. G.; JENSEN, H. H. Reliability and validity of nutrition knowledge and diet-health awareness tests developed from the 1989- 1991 Diet and Health Knowledge Survey. Journal of Nutrition Education and Behavior, v. 29, n. 2, p. 63-72, mar. 1997.

SCAGLIUSI, F. B.; POLACOW, V. O.; CORDÁS, T. A.; COELHO, D.; ALVARENGA, M.; PHILIPPI, S. T.; LANCHA JR., A. H. Tradução, adaptação e avaliação psicométrica da Escala de Conhecimento Nutricional do National Interview Survey Cancer Epidemiology. Revista de Nutrição, v. 19, n. 4, p. 425-436, jul.-ago. 2006.

WORLD HEALTH ORGANIZATION (WHO). Defining the problem of overweight and obesity. In: WORLD HEALTH ORGANIZATION. Obesity: preventing and managing the global epidemic: report of a WHO Consultation. Geneva, 2000.

ZAWILA L. G.; STEIB, C. S.; HOOGENBOOM, B. The female collegiate cross-country runner: nutritional knowledge and attitudes. Journal of Athletic Training, v. 38, n. I. p. 64-74, mar. 2003.

Recebido: 13 set. 2009 Aprovado: 30 maio 2010

Endereço para correspondência:

Murilo Dáttilo

Rua Giestas, 63, ap. 62a - Vila Bela

São Paulo-SP

CEP 03। 47-000 